\title{
Pellagra encephalopathy among tuberculous patients: its relation to isoniazid therapy
}

\author{
NOBUYOSHI ISHII, YASUO NISHIHARA
}

From the Department of Pathology, University of Occupational and Environmental Health, Kitakyushushi, and the Department of Medicine, Kurate Kyoritsu Hospital, Miyatacho, Fukuokaken, Japan

SUMMARY Eight cases of pellagra, diagnosed on the grounds of neuropathological findings and retrospective study of clinical data, were found among 106 necropsy cases of tuberculosis. Although these eight patients had shown various mental, neurological and gastrointestinal symptoms, as well as skin lesions, the diagnosis of pellagra had not been made clinically. In all the patients, pellagra symptoms appeared during isoniazid therapy. Death occurred 4 to 16 weeks later. Isoniazid inhibits the conversion of tryptophan to niacin and may induce pellagra, particularly in poorly nourished patients. Pellagra should be suspected whenever tuberculous patients under treatment with isoniazid develop mental, neurological or gastrointestinal symptoms, even in the absence of typical pellagra dermatitis.

Pellagra is a disease caused primarily by niacin (nicotinic acid) deficiency and characterised by the classical triad of dermatitis, diarrhoea and dementia. A niacin deficiency state can be brought about by various causes, such as inadequate food intake, malabsorption syndrome, increased requirement or administration of drugs which interfere with niacin synthesis. Tuberculous patients may develop pellagra, owing to an increase in niacin requirement and isonicotinic acid hydrazide (isoniazid) therapy. Isoniazid is a widely used, highly effective antituberculous drug. It has a structural formula similar to niacin. Isoniazid interferes with the conversion of tryptophan to niacin, by producing a deficiency in the pyridoxine coenzymes required for the conversion. Therefore, isoniazid may induce various manifestations of pellagra, particularly in poorly nourished patients. There are several reports of isoniazid-induced pellagra, based on clinical grounds; ${ }^{1-6}$ however, to our knowledge, no necropsy study has been published.

We found eight cases showing neuropathological features of pellagra among 106 necropsy cases of tuberculous patients. These eight patients had pre-

Address for reprint requests: Dr N Ishii, Department of Pathology (Neuropathology), University of Occupational and Environmental Health, School of Medicine, Iseigaoka 1-1, Yahatanishiku, Kitakyushushi, Fukuokaken, 807, Japan.

Received 24 July 1984 and in revised form 6 November 1984. Accepted 10 November 1984 sented with various mental, neurological, gastrointestinal and skin lesions during the course of isoniazid therapy. Clinically, pellagra had not been suspected in any of them. The aim of this paper is to show the clinical and pathological findings of the eight cases and to call attention to pellagra encephalopathy without skin lesions (pellagra sine pelle agra).

\section{Cases and methods}

From 1965 to 1984,4551 patients were admitted to Kurate Kyoritsu Hospital. Of these, 1437 died and complete post-mortem examination was done on 1124 cases. There were 106 cases of pulmonary tuberculosis. Seventy cases had been treated for tuberculosis until shortly before death. The other 36 cases had not been treated, because the tuberculous lesions were old or were identified only at post-mortem examination. All the organs including the brain were kept in $10 \%$ formalin for 2 weeks and blocks were taken from each organ. Regions examined were frontal lobe (areas 4 and 8), parietal and occipital lobes, Ammon's horn and inferior temporal region, basal ganglia, thalamus, midbrain, pons, medulla, cerebellar cortex and dentate nucleus. Sections were stained with haematoxylin and eosin. In the eight cases of pellagra, Nissl, Bodian and periodic-acid-Schiff stains were also done. Clinical data were obtained from the charts. Representative cases are described below.

Case 1 In October 1965, the patient, a man then aged 48 years, was found to have pulmonary tuberculosis. He had been an alcoholic for about 15 years, and had had alcoholic gastritis and liver dysfunction several times, but there was 
no past history of alcoholic psychosis nor any other mental disease. He abstained from drinking and started to take isoniazid $0.4 \mathrm{~g}$ daily, p-amino-salicylic acid (PAS) $10 \mathrm{~g}$ daily and streptomycin $1.0 \mathrm{~g}$ im twice weekly, at a tuberculous sanatorium as an out-patient. In June 1967 he began to show a disturbance of gait and was admitted to the sanatorium on 15 August. By mid-September he became confined to bed with paraparesis and incontinence of both urine and faeces, and showed evidence of mental disturbance. He was transferred to Kurate Kyoritsu Hospital from the sanatorium on 26 September, 1967 with a diagnosis of acute catatonic schizophrenia. On admission he was confused and disoriented as to time and place. General physical examination was unremarkable, except for marked emaciation. Blood pressure was $142 / 92 \mathrm{~mm} \mathrm{Hg}$ and pulse rate 96 per minute. No skin lesions suggestive of pellagra were noted. Neurological examination disclosed facial myoclonus, paraparesis with marked spasticity and bilateral ankle clonus. There was slight anaemia. Other laboratory data were all within normal limits. Lumbar puncture revealed normal cerebro-spinal fluid. Isoniazid was withdrawn from 26 September, however he continued to have insomnia, visual and auditory hallucinations. He muttered to himself and moved his hands all the time, as though he was seeing something in front of him. His delirious state persisted with fluctuation of the level of consciousness. Generalised seizure was observed twice. He became dysphagic and died of bronchopneumonia on 7 October, 1967 aged 50 years.

Necropsy revealed bilateral aspiration pneumonia and fibrinocaseous tuberculosis in the right upper lobe. The brain weighed $1420 \mathrm{~g}$. Grossly, no abnormality was noted. Histologically there was marked central chromatolysis of neurons occurring over wide areas. The Betz cells and other relatively large pyramidal neurons of the cerebral cortex, as well as the neurons of the pontine nuclei, perihypoglossal nuclei (nucleus of Roller), arcuate nuclei and dentate nuclei of the cerebellum showed severe central chromatolytic changes (figs 1 and 2). No other histopathological changes were noted in the brain. A section of the tongue revealed slight parakeratosis, acanthosis and chronic inflammatory cell infiltrates in the submucosa.

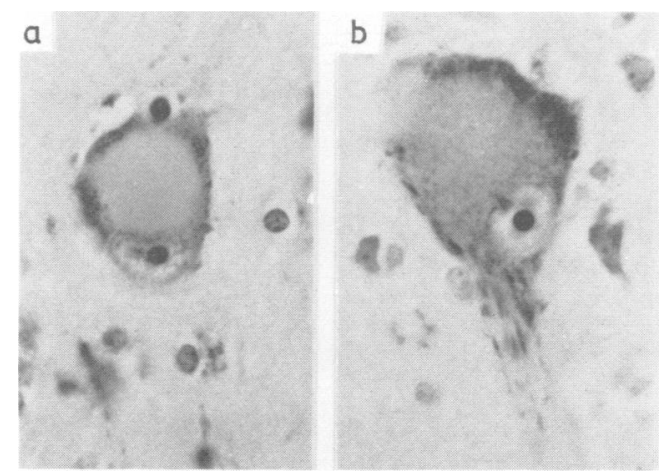

Fig 1 Central chromatolysis in Betz cells. Nissl $\times 400$, case 1 .

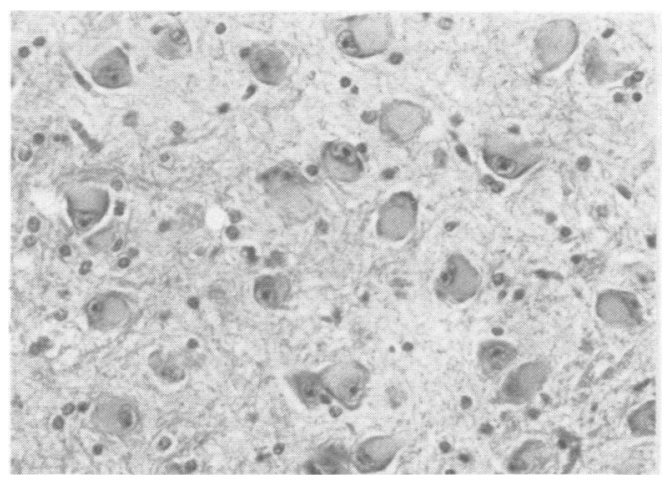

Fig 2 Central chromatolysis in neurons of pontine nuclei. $H \& E \times 200$, case 1 .

Case 2 A man, aged 21 years, was found to have pulmonary tuberculosis and was admitted to a local hospital. He was treated with isoniazid $0.4 \mathrm{~g}$ and PAS $10 \mathrm{~g}$ daily. About a year later, he started to smile for no apparent reason and mutter to himself. He refused to eat or take any medication. He was sent to a mental institution where a diagnosis of schizophrenia was made. His condition and treatment for the next 2 years are not known to us. In September 1960 , he was discharged and remained at home for one and a half years, taking only isoniazid. In March 1962, he entered Kurate Kyoritsu Hospital, because his pulmonary condition had worsened and the sputum was positive for tuberculous bacilli. Treatment was begun with isoniazid $0.4 \mathrm{~g}$ and PAS $10 \mathrm{~g}$ daily, and streptomycin $1.0 \mathrm{~g}$ im twice a week. For the next 4 years he was treated with several other antituberculous drugs such as sulfamethoxazole, ethambutol, ethionamide, kanamycin and cycloserine, as well as isoniazid, PAS and streptomycin in various combinations. During the 4 year period he had been indifferent to his surroundings and occasionally carried on a monologue and wore an inappropriate smile, for which chlorpromazine, perphenazine and minor tranquillisers were prescribed. Cycloserine was administered for 1 month in 1965 and 2 months in 1967, during which time there were no changes in his mental symptoms. Since October 1967, his treatment consisted of isoniazid $0.4 \mathrm{~g}$, ethionamide $0.3 \mathrm{~g}$ and ethambutol $1.0 \mathrm{~g}$ per day. In early August 1968 , he developed sunburn-like skin lesions on hands and feet. By late August they became typical symmetrical pellagrous dermatitis with Casal's necklace and dyssebacia on the face. He was depressed and apathetic. All the antituberculous drugs were discontinued for 1 month. From 26 August, multi-vitamins including niacin, $B_{1}, B_{2}, B_{6}, B_{12}$ were prescribed. The skin lesions and mental symptoms cleared by 10 September. After this episode his attending physician changed twice. He again started to take isoniazid $0.4 \mathrm{~g}$ and sulfamethoxazole $1.0 \mathrm{~g}$ per day. In July 1970 he developed eczema-like lesions on the face and in early August symmetrical dermatitis appeared on hands, feet, face and neck. He also had diarrhoea, anorexia, vomiting, insomnia and gait disturbance with spasticity of the legs. This time vitamin $B_{1}, B_{6}$ and $B_{12}$ were given, but niacin was not prescribed, because the physician in charge did not 
suspect pellagra. He expired on 10 October, 1970 . Necropsy revealed old tuberculous lesions in both upper lobes. The brain weighed 1360 grams. Histological findings were similar to that of case 1 , showing severe central chromatolysis of neurons in many areas. Pathologically there was no immediate cause of death, other than pellagra.

The clinical and some pathological findings of the eight cases are summarised in the table.

Summary of the clinical findings All the patients were male. Their age ranged from 35 to 72 years at the time of death, with a mean of 51 years. The total course of tuberculosis extended from 1 year and 2 months to 13 years. Six of the eight patients were transferred to Kurate Kyoritsu Hospital from four different sanatoria after pellagra psychosis occurred, because the hospital had wards for both tuberculosis and psychiatry. The periods of hospitalisation until demise were 3,6 and 9 days, 7,9 and 14 weeks, 15 months and 8 years. They expired between 1965 and 1972, five of them before 1967.

Antituberculous drugs given during the courses varied from case to case. But to all of them $0.4 \mathrm{~g}$ of isoniazid was administered as a first choice, along with PAS and streptomycin. PAS was given $10 \mathrm{~g}$ daily and streptomycin $1.0 \mathrm{~g}$ im twice weekly. Other antituberculous drugs administered were sulfamethoxazole $1.0 \mathrm{~g}$ daily ( 4 cases), ethionamide $0.3-0.5 \mathrm{~g}$ daily (3 cases), ethambutol $0.75-$ $1.0 \mathrm{~g}$ daily ( 3 cases), kanamycin $1.0 \mathrm{~g}$ im twice a week ( 3 cases) and cycloserine $0.5 \mathrm{~g}$ daily ( 1 case). Cycloserine was administered in case 2 only for short periods. Although these drugs were prescribed in various combinations, isoniazid had been administered in all cases before the onset of pellagra symptoms.

Early mental symptoms observed were insomnia (8/8) and anxiety $(6 / 8)$. followed by confusion $(8 / 8)$, restless behaviour $(5 / 8)$, monologue $(7 / 8)$, inappropriate smile $(4 / 8)$ and refusal of nourishment $(5 / 8)$. Terminally hallucinations (6/8) and a delirious state with fluctuation of the level of consciousness (4/8) were noted. Depression, neurasthenia and apathy were recorded in some cases. Case 3 attempted suicide while in a confused state 6 days prior to death. A clinical diagnosis of schizophrenia was made in four cases (case 1,2,3, and 5) on account of these mental symptoms. Cases 4 and 8 were diagnosed as

Table Clinical and pathological findings of 8 cases of pellagra

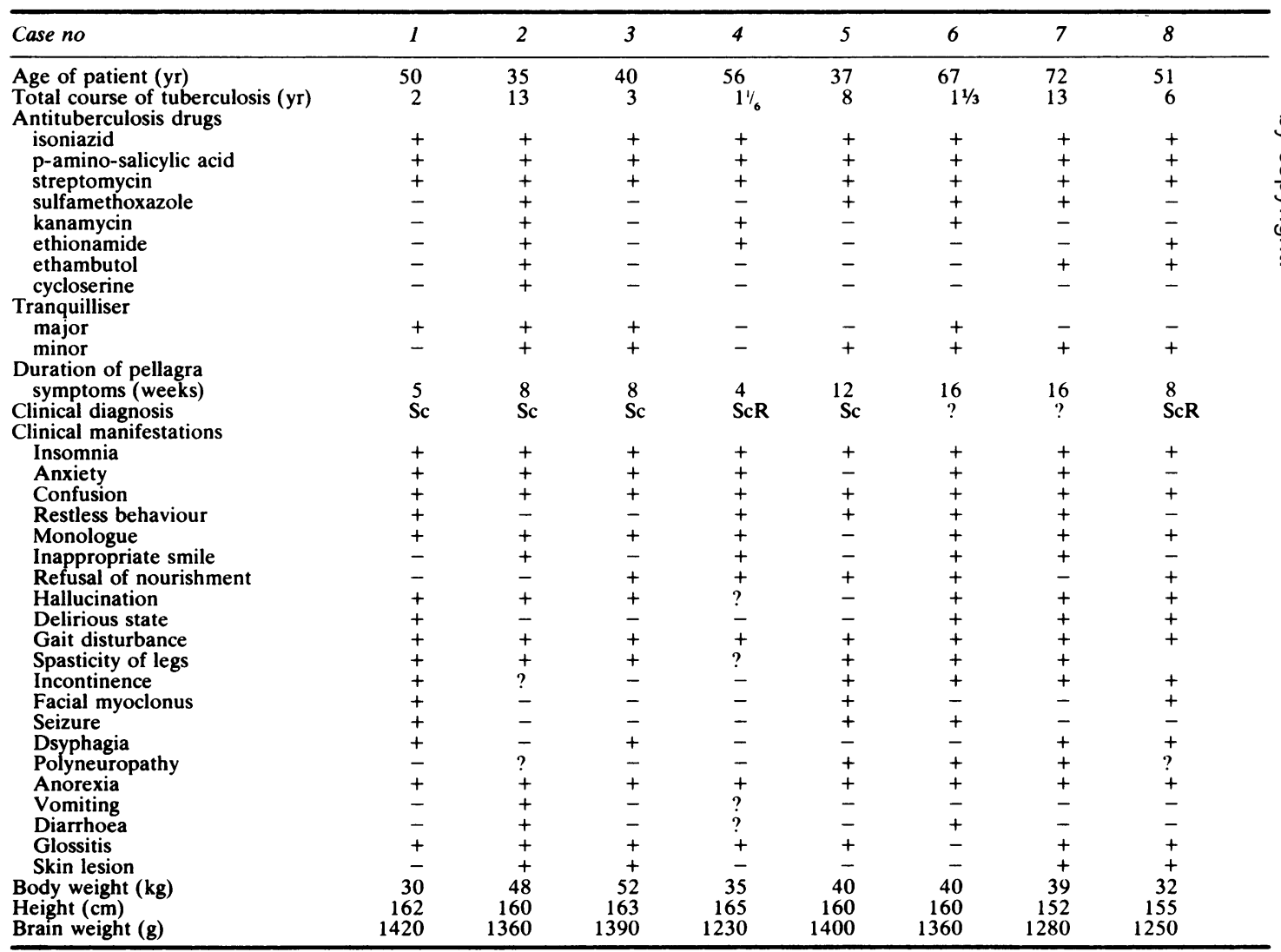

Sc: Schizophrenia, ScR: Schizophrenic reaction, ?: Not certain. 
schizophrenic reaction. Case 6 was diagnosed as depressive reaction, probably associated with cerebral arteriosclerosis, and case 7 as encephalopathy of unknown cause, possibly due to arteriosclerosis. The exact cause of mental symptoms of cases 2 and 6 prior to the onset of pellagra is not known. It cannot be ruled out that case 2 might have had schizophrenia as well. For these mental symptoms of pellagra, various major and minor tranquillisers were given, such as chlorpromazine, levomeprovazine, perphenazine, haloperidol, diazepam, nitrazepam, chlordiazepoxide and phenobarbital, but without much benefit.

Neurological symptoms recorded on the charts were gait disturbance $(8 / 8)$, spasticity of the legs with hyperreflexia $(7 / 8)$, incontinence of both urine and faeces $(5 / 8)$, facial myoclonus $(3 / 8)$, focal and generalised seizures $(3 / 8)$, dysphagia (4/8), and polyneuropathy (3/8). Gastrointestinal symptoms were anorexia $(8 / 8)$, diarrhoea $(2 / 8)$, nausea and vomiting (1/8). Anorexia was usually seen prior to the onset of terminal pellagra symptoms.

Skin lesions were observed in four cases. Case 2 presented typical symmetrical pellagra dermatitis on the exposed parts of the body. On the first occasion, it was cured by multi-vitamin tablets including niacin. But on the second episode of pellagra dermatitis, which resulted in the patient's death because pellagra was not suspected clinically, no treatment was undertaken. In case 3 the skin pigmentation was thought to be due to Addison's disease by an attending physician. At necropsy there was no adrenal abnormality. In case 7 , facial lesions were described as dyssebacia and eczema-like dermatitis on the chart. These lesions were possibly features of pellagra dermatitis, because they are seen in those who have been bedridden for a period of time. In case 8, pigmentation, rough and scally lesions were noted.

Laboratory data examined in the terminal stage of the illness were insufficient. However, there were indications of malnutrition. Anaemia (a haemoglobin below 70\%) was seen in six cases. Four cases had hypoproteinaemia (serum protein less than $6.0 \mathrm{~g} / \mathrm{dl}$ ). Lumbar puncture was performed in two cases, on account of possible tuberculous meningitis, but failed to reveal any abnormality. In one case a liver function test showed slight elevation of SGOT and SGPT. After the occurrence of terminal pellagra, treatment comprised intravenous administration of glucose, electrolytes and vitamins. Vitamin $B_{1}$ was given in all cases, $B_{2}$ in 4 cases, $B_{6}$ in 2 cases, $B_{12}$ in 2 cases and $C$ in five cases.

The clinical features of the eight cases have many similarities. These patients had been treated for tuberculosis for the periods of 1 to 13 years with various drugs. They had anorexia and were emaciated, but had been taking $0.4 \mathrm{~g}$ of isoniazid daily. They developed various mental symptoms for which diagnosis of schizophrenia or schizophrenic reaction was made. Subsequently, neurological signs such as spasticity and double incontinence appeared. Summary of the necropsy findings Malnutrition was obvious in all cases. Body weights at necropsy ranged from 30 to $52 \mathrm{~kg}$, with an average of $39.5 \mathrm{~kg}$. The brains showed no significant macroscopic abnormalities. Cases 6 and 7 exhibited slight and moderate arteriosclerotic changes. But there were no vascular lesions within the cerebral parenchyma. Histologically the most striking and constant

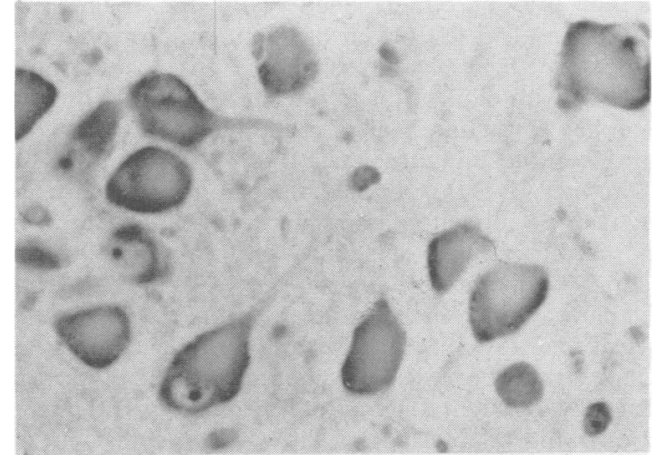

Fig 3 Central chromatolysis in neurons of dorsal vagal nucleus. Nissl $\times 300$, case 8 .

finding was central chromatolysis of neurons. The chromatolytic change was observed in Betz cells (8/8), and othe relatively large cortical neurons $(8 / 8)$, neurons of pontine nuclei $(8 / 8)$, gracile and cuneate nuclei $(8 / 8)$, descending trigeminal nuclei $(8 / 8)$, arcuate nuclei $(8 / 8)$, vestibular nuclei $(8 / 8)$, dentate nuclei $(8 / 8)$, perihypoglossal nuclei (nuclei of Roller) (7/8), dorsal vagal nuclei (6/8) and anterior horn cells of the upper cervical cord (2/2) (fig 3). A few neurons in the thalamus and basal ganglia exhibited similar chromatolytic changes. No chromatolysis was seen in the Purkinje cells, the neurons of substantia nigra, Ammon's horn and hypoglossal nuclei, although among the largest neurons. There were no lesions suggestive of Wernicke's disease, cerebellar cortical degeneration, central pontine myelinolysis or other disorders due to malnutrition. Histological features of tuberculous meningitis

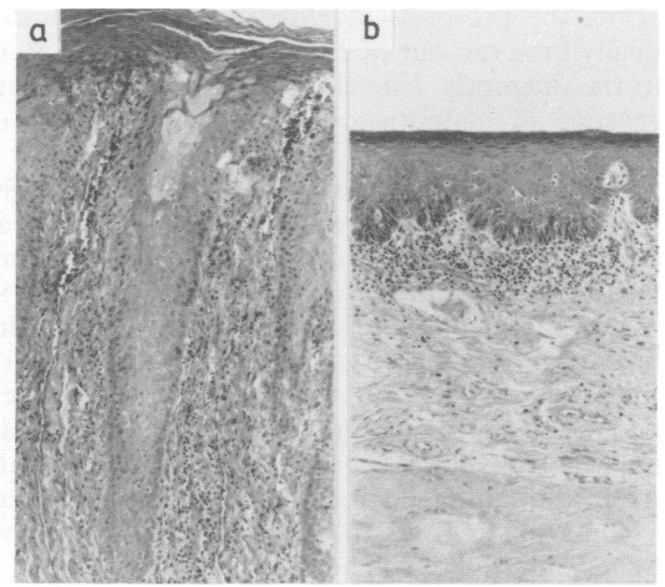

Fig 4 (a) Glossitis showing parakeratosis, elongation of rete ridges and chronic inflammatory cell infiltrates. $H$ \& $E \times 75$, case 3. (b) Glossitis showing atrophy of the squamous epithelium and chronic infiammatory cell infiltrates. $H \& E \times 75$, case 6 . 
were not present either. Sections of the tongue were examined in all cases. Seven of them demonstrated slight to moderate degrees of glossitis, such as parakeratosis, acanthosis with elongation of rete ridges or atrophy of the squamous epithelium, and chronic inflammatory cell infiltrates in the submucosa (fig 4).

Pathologically, the immediate cause of death was thought to be bronchopneumonia in six cases. But in two cases the direct cause of death was uncertain, other than pellagra. The livers were unremarkable, except for case 8 , which clinically had increased transaminase, exhibited histological features consistent with drug induced hepatitis.

Among the 106 cases of tuberculosis, there were five others showing slight to moderate chromatolytic changes in Betz cells and pontine nuclei. However, they did not present any clinical features suggestive of pellagra, such as mental, neurological and gastrointestinal symptoms or skin lesions. Therefore, they were excluded from the present pellagra group. Two of them were patients with senile dementia of Alzheimer type.

\section{Discussion}

Only four of these eight cases had skin lesions which were highly suggestive of pellagra dermatitis, but were not so diagnosed. None had the diagnosis confirmed by biochemical methods (excretion of $\mathrm{N}^{\prime}$-methylnicotinamide etc). However, we are convinced that these eight cases had "pellagra" on the following neuropathological and clinical grounds. The most consistent neuropathological change in pellagra is said to be central chromatolysis. The distribution of the chromatolytic neurons in our eight cases was similar to those reported by many authors. ${ }^{7-11}$ Although the histological picture of pellagra glossitis (as of pellagra dermatitis) is nonspecific, the presence of glossitis confirmed histologically in seven out of eight cases is in agreement with the diagnosis. Emaciation and laboratory data suggestive of malnutrition were also in favour of pellagra.

. The mental symptoms observed in the eight patients were insomnia, anxiety, depression, neurasthenia, apathy, confusion, hallucination and delirium. All of these symptoms may occur in endemic and alcoholic pellagrins. ${ }^{7-16}$ However, the mental symptoms were considered to be those of schizophrenia or schizophrenic reaction in six cases. McConnell and Cheetham ${ }^{1}$ reported a 22-year-old male who developed acute pellagra with skin, gastrointestinal and various mental symptoms during isoniazid therapy. A psychiatrist who saw this patient reported that he was suffering from a schizophrenic reaction which presented many catatonic features. Shah ${ }^{13}$ observed various psychotic manifestations in 19 cases among the 22 pellagrins with skin lesions in India. Two of them presented acute schizophreniform psychosis with acute catatonic excitement, disorientation, hallucination, delusion etc.

Neurological symptoms of pellagra are also characteristic. ${ }^{17}$ Leigh $^{10}$ collected 14 necropsy cases of pellagra, among whom spastic paraplegia was noted in three cases. We reported 20 necropsy cases of alcoholic pellagra. ${ }^{\text {s }}$ Gait disturbance, extrapyramidal rigidity and hyperreflexia of the legs, incontinence of urine and faeces were observed in the majority of the cases. Polyneuropathy and seizure were also noted in some cases. Jolliffe et al ${ }^{16}$ listed cogwheel rigidity of the extremities as one of the characteristic neurological features. Our present eight cases showed gait disturbance, spasticity of the legs and incontinence in almost all the cases, as well as polyneuropathy, facial myoclonus, seizure and dysphagia in some cases. These neurological symptoms are not diagnostic of, but are consistent with pellagra.

Isoniazid has a structural formula similar to niacin and nicotinamide. Nicotinamide functions as the component of two important coenzymes, NAD (nicotinamide adenine dinucleotide) and NADP (nicotinamide adenine dinucleotide phosphate), which are functional groups in intracellular oxidation reduction enzyme systems necessary for tissue respiration, glycolysis, and fat synthesis. In nicotinamide deficiency states, such as poorly nourished patients or chronic tuberculous patients, cells may incorporate the structurally similar isoniazid for the formation of NAD and NADP which then does not function as a coenzyme and may lead to the development of pellagra. ${ }^{5}$ But this theory is not likely. It is known that isoniazid intake produces pyridoxine deficiency which leads to a disturbance of normal tryptophan metabolism to niacin. $^{18}$

There are several case reports of isoniazidinduced pellagra..$^{1-6}$ In all of these cases pellagra dermatitis is described. Some demonstrate mental and neurological symptoms, such as confusional psychosis, schizophrenic reaction, increased tone in the legs. On the other hand, cases are reported of isoniazid-induced encephalopathy. ${ }^{19-25}$ Their mental and neurological symptoms are described as insomnia, anxiety, depression, disorientation, hallucination, acute toxic confusional psychosis, schizoid reaction, increased muscle tone, spastic paraplegia, incontinence etc. Skin lesions are not mentioned. Clinical features of our eight cases resemble closely those of isoniazid-induced pellagra and encephalopathy. The reported cases of isoniazid-induced pellagra showed a good response to niacin or nicotinamide administration. Most cases of isoniazid-induced encephalopathy were not treated with niacin. However, $\mathrm{Zabad}^{20}$ described a 
case of confusional psychosis without pellagra dermatitis who responded well to nicotinamide. Wood ${ }^{3}$ reported three cases of isoniazid-induced confusional psychosis without dermatitis, who showed dramatic, good and fair response to niacin treatment respectively. Jackson's two cases also recovered with niacin treatment. Haynes' first case is worth mentioning. ${ }^{5}$ A 17-year-old girl developed mental confusion, apathy and anorexia after 6 months of hospital treatment with isoniazid and PAS for pulmonary tuberculosis. On allowing the patient to get up and about, a typical pellagrous rash appeared 7 weeks after the onset of mental symptoms. Pellagrous dermatitis is a photosensitive lesion and appears on the exposed parts of the body. Therefore, the pellagrins who have been bedridden for a period of time do not develop typical skin lesions. This stage of pellagra psychosis without skin lesion is called pellagra sine pelle agra or pellagra ante pellagram, which has been known for nearly two hundred years. Haynes ${ }^{5}$ mentioned on his clinical observation alone that toxic psychosis, which seemed to occur among hospital patients with isoniazid therapy, might possibly be attributed to nicotinamide deficiency but this question required further investigation.

All of our eight patients had been in the hospital when they developed pellagra psychosis. They probably did not have much chance to be exposed to the sunlight. Four of our eight cases did not have skin lesions. But neuropathologically they showed severe chromatolytic neurons in the same distribution as in the other four cases. So we believe they are cases of pellagra psychosis without skin lesions, namely pellagra sine pelle agra. We have no experience of isoniazid-induced encephalopathy or toxic confusional psychosis, being treated successfully with niacin. But we think that at least some of these cases had pellagra psychosis. Shiozaki et al ${ }^{26}$ reported in 1954 that the incidence of schizophrenia had increased among tuberculous patients since the advent of isoniazid. We would like to emphasise that one should administer niacin, pyridoxin and other vitamins to those patients with mental symptoms and study the response.

Before the advent of isoniazid, Ying-K' uei ${ }^{y}$ noticed that diarrhoea, tuberculosis and malnutrition were the outstanding clinical features among the necropsy cases of pellagra. Biehl and Vilter ${ }^{27}$ found that, with daily doses of $5 \mathrm{mg}$ per $\mathrm{kg}$ body weight, neurological side-effects occurred in only $2 \%$ of patients, but with a daily dose of $6-10 \mathrm{mg}$ per $\mathrm{kg}$ the incidence rose to $10 \%$ and with $11-15 \mathrm{mg}$ it went up to $20 \%$. Our eight cases had tuberculosis, malnutrition and overdose of isoniazid as compared with their body weight. The isoniazid daily doses ranged from $7.7 \mathrm{mg}$ to $13.3 \mathrm{mg}$ per $\mathrm{kg}$. Case $2 \mathrm{had}$ had ethionamide $0.3 \mathrm{~g}$ per day along with isoniazid, prior to the first episode of pellagra. Ethionamide (ethyl-thio-isonicotinamide) is a derivative of isoniazid and might also inhibit niacin synthesis.

Pellagra is now considered to be a disappearing disease, particularly in the developed countries. However, the disease is still occasionally observed among chronic alcoholics, food faddists, patients with malabsorptive disease of the gastrointestinal tract and with malignant carcinoid. It may also be seen in patients treated with chemotherapeutic agents which inhibit tryptophan metabolism or antagonise one of the vitamins associated with this pathway, such as isoniazid, thiosemicarbasone derivatives, 6-Mercaptopurine, 5-fluorouracil and puromycin. ${ }^{28}$ Still" ${ }^{1}$ wrote that pellagra sine pelle agra still lies hidden and unsuspected in the mortality data of mental hospitals. We should not forget pellagra, an easily curable disease, in such patients.

\section{References}

' McConnell RB, Cheetham HD. Acute pellagra during isoniazid therapy. Lancet 1952;ii:959-60.

2 Jones WA, Jones GP. Peripheral neuropathy due to isoniazid; Report of two cases. Lancet 1953;i: 1073-4.

${ }^{3}$ Wood MM. Central nervous system complications during INH treatment of pulmonary tuberculosis. $\mathrm{Br} J$ Tuberculosis 1955;1:20-9.

${ }^{4}$ Harrison RJ, Feiwel M. Pellagra caused by isoniazid. $\mathrm{Br}$ Med J 1956;II:852-4.

${ }^{5}$ Haynes WS. Pellagra occurring in Africans under treatment for pulmonary tuberculosis. East African Med $J$ 1958;35: 171-80.

- Shankar PS. Pellagra in Gulbarga. J Indian Med Ass 1970;54:73-5.

7 Langworthy OR. Lesions of the central nervous system characteristic of pellagra. Brain 1931;54:291-302.

${ }^{8}$ Greenfield JG, Holmes JM. A case of pellagra; The pathological changes in the spinal cord. $\mathrm{Br}$ Med $J$ 1939;i:815-9.

${ }^{y}$ Ying-K'uei H. Pathologic anatomy of human nervous system in avitaminosis. Arch Neurol Psychiatr 1942;48:271-319.

${ }^{10}$ Leigh D. Pellagra and the nutritional neuropathies; A neuropathological review. J Ment Sci 1952;98:13042.

"Still CN. Nicotinic acid and nicotinamide deficiency: Pellagra and related disorders of the nervous system. In: Vinken PJ, Bruyn GW, eds. Handbook of Clinical Neurology vol 28. Amsterdam: North-Holland, 1975:59-104.

${ }^{12}$ Spies TD, Aring CD, Gelperin J, Bean WB. The mental symptoms of pellagra. Their relief with nicotinic acid. Am J Med Sci 1938;196:461-75.

${ }^{13}$ Shah DR, Pandey SK, Rathi R. Psychiatric manifestation in pellagra. J Ass Physicians India 1972;20:5758.

14 Klauder JV, Winkelman NW. Pellagra among chronic alcoholic addicts. JAMA 1928;90:364-71. 
15 Ishii N, Nishihara Y. Pellagra among chronic alcoholics: Clinical and pathological study of 20 necropsy cases. $J$ Neurol Neurosurg Psychiatry 1981;44:209-15.

16 Jolliffe N, Bowman KM, Rosenblum LA, Fein HD. Nicotinic acid deficiency encephalopathy. JAMA 1940;114:307-12.

17 Shah DR, Singh SV, Jain IL. Neurological manifestations in pellagra. $J$ Ass Physicians India 1971;19:443-6.

${ }^{18}$ McIlwain H, Bachelard HS. Biochemistry and the Central Nervous System. 4th ed. London: Churchill Livingstone, 1971:284.

${ }^{19}$ Hunter RA. Confusional psychosis with residual organic cerebral impairment following isoniazid therapy. Lancet 1952;ii:960-2.

${ }^{20}$ Zabad M. Psychosis with isoniazid therapy. Lancet 1953;i: 293.

${ }^{21}$ Biehl JP, Nimitz HJ. Studies on the use of a high dose of isoniazid; Toxicity studies. Am Rev Tuberculosis
1954;70:430-41.

${ }^{22}$ Jackson SLO. Psychosis due to isoniazid. $\mathrm{Br}$ Med $\mathrm{J}$ 1957;ii: 743-6.

${ }^{23}$ Hare EH. Psychotic reactions due to antituberculous drugs. Tubercle 1958;39:90-5.

${ }^{24}$ Adams BG, Davis BM. Neurological changes associated with PAS and INAH therapy. J Ment Sci 1961;107:943-7.

${ }^{25}$ Adams P, White C. Isoniazid-induced encephalopathy. Lancet 1965;i:680-2.

${ }^{26}$ Shiozaki M. Psycho-neurological symptoms of tuberculous patients treated with isonicotinic acid hydrazine. Iryo $1954 ; 8: 25-30$.

${ }^{27}$ Biehl JP, Vilter RW. Effects of isoniazid on pyridoxin metabolism. JAMA 1954;156:1549-52.

${ }^{28}$ Barrett-Connor $E$. The etiology of pellagra and its significance for modern medicine. Am J Med 1967;42:859-67. 